\title{
Sparse Converter Placement in WDM Networks and their Dynamic Operation Using Path-Metric Based Algorithms
}

\author{
Sanjay K. Bose, SMIEEE, Y.N. Singh, MIEEE \\ Department of Electrical Engineering \\ Indian Institute of Technology \\ Kanpur - 208 016, INDIA
}

\author{
A.N.V.B. Raju \\ Samsung India \\ \#67, Infantry Road \\ Bangalore - 560 001, INDIA
}

\author{
Bhoomika Popat \\ Jagdish Bhawan, BRBRAITT \\ Ridge Road \\ Jabalpur - 482 001, INDIA
}

\begin{abstract}
We consider WDM networks with lightpath switching where wavelengths may be converted, as required, along the lightpath. For efficient converter usage in such a network, sparse converter placement may be followed where only some of the network nodes are equipped with wavelength converters. Given the nominal network traffic pattern, we present a simple heuristic algorithm, which may be used to determine the location of these converters for good network performance. For a network designed in this fashion, we consider the application of a path-metric based heuristic algorithm for lightpath routing and wavelength selection along the links of the selected route. Dynamic operation of the sparse converter network is considered using this path-metric based algorithm for lightpath routing and wavelength selection.
\end{abstract}

\section{INTRODUCTION}

Wavelength Division Multiplexed (WDM) all-optical networks, with several optical wavelengths multiplexed on individual fibers, are expected to provide the communications resources for both long and short-haul networks in the near future [1]. In these networks, the end-users are expected to establish lightpaths between themselves for their communication requirements. A lightpath may either be wavelength continuous (WC) where the same optical wavelength is used in all its links or it may be nonwavelength continuous (NWC) where the wavelengths may be modified as required/desired at the intermediate nodes of the path. A WC lightpath may undergo space switching at the intermediate nodes but no wavelength conversion whereas a NWC lightpath may also require the latter at some nodes. Various issues related to wavelength conversion in WDM networks are presented in [2],[3]. Some simple heuristic algorithms for lightpath routing and wavelength assignment are considered in [4].

Given the current costs and technological limitations of wavelength converters, various kinds of limited or partial wavelength conversion have been proposed. This comprises of (a) limited number of converters at the nodes (limited wavelength conversion), (b) limited number of nodes equipped with converters (sparse conversion), or (c) limited range of wavelengths to which an input wavelength may be converted (partial or limited range conversion). Combinations of these may also be implemented. For nodes with a limited number of converters, the converters may be shared either on a share-per-node or share-per-link [2] basis. While (c) may arise because of technological limitations, (a) and (b) are really motivated by the fact that only a few converters may actually be needed in a network. Reducing the number of converters by following these two approaches would then lead to higher overall converter usage.

Given this context of WDM networks with limited/partial conversion, this paper examines two related topics. Firstly, in Section II, we propose a simple heuristic algorithm to guide us in deciding the network nodes that should be equipped with converters. This is done for a given value of the nominal traffic load pattern offered to the network assuming full conversion at the converter nodes and is shown to be close to what may be obtained from a rigorous optimization process. Secondly, we propose in Section III another heuristic algorithm based on path-metric calculations, which may be used for dynamic operation of the network. This may be used to do lightpath routing and wavelength selection in a dynamic fashion. In Section IV, we apply this path-metric based algorithm to operate a sparse-converter WDM network and study its performance. Section V concludes the paper.

\section{Converter Placement in Sparse Conversion NETWORKS}

Sparse wavelength conversion, with only a few of the nodes equipped with converters, offers a good trade-off between the cost of the converters and the performance improvement obtained by their incorporation in a WDM network. Earlier efforts in this area [5]-[7] have been concerned with the optimal placement of converter nodes in the network. The disadvantage of such optimal algorithms is their complexity, especially when considering large networks. An attempt to simplify this by using a good heuristic algorithm is considered in [8]. The disadvantage of the heuristic algorithm of [8] is that it proceeds by adding one converter node at a time to the system, thereby making the algorithm slow and difficult to use for large networks. It is also not clear that this gradual increase in the number of convertible nodes will indeed give a result close to the optimal in the case of large networks. A genetic algorithm based approach has also been tried in [9]. Based on our work [10], we propose a simple heuristic algorithm for converter placement in this paper. This is shown to provide nearoptimal results for a wide variety of networks and traffic loading scenarios.

We consider a network with $N$ nodes. The links of the network are assumed to be bi-directional with one fiber for each direction. We assume $W$ wavelengths for each fiber where a lightpath requires one wavelength on each link that it traverses in going from the source to the destination. New lightpath requests for a given source-destination pair $(s, d)$ are 
assumed to come from a Poisson process with mean rate $\lambda_{s d}$ $\left(\lambda_{s d}=0\right.$ for $\left.s=d\right)$ and have holding times which are exponentially distributed with a mean of unity. This latter assumption implies that the mean holding times of all the lightpaths are the same and that the time-scale is normalized to this quantity. Note that $\lambda_{s d}$ may then also be considered as the lightpath traffic from node $s$ to $d$.

Given the network graph, the nominal traffic loading pattern $\left\{\lambda_{i j}\right\} \quad i, j=1, \ldots, N$ and the number of nodes $K$ where converters are to be placed, the problem addressed by our heuristic algorithm is to identify the $K$ nodes (out of $N$ ) which are to be equipped with wavelength converters. (We assume full conversion capability at the selected nodes but do consider operation with limited number of converters in the next section.) Our proposed heuristic does this by evaluating a weighting factor $x(i)$ for the $i^{\text {th }}$ node which is indicative of the desirability of placing converters at that node.

In order to estimate $x(i)$, we assume that Djikstra's shortest path algorithm is used to find the nominal lightpath routing between each node pair. (During actual dynamic operation, as considered in Sec. III, other alternate routes may also be considered.) The converter placement heuristic may also be extended to allow for multiple routing choices. However, this will be more complex and has not been presented here. Let $H(s, d)$ be the number of hops in the shortest path between the source-destination nodes $s$ and $d$. Let $L(s, d)$ be the number of other (shortest) paths between other node-pairs which share one or more links with the shortest path from $s$ to $d$. We index these paths using $j$ ranging from 1 to $L(s, d)$ and let $n(s, d, j)$ be the number of links common between the $j^{\text {th }}$ such path and the shortest path from $s$ to $d$. The mean interference length $l(s, d)$ may then be defined as

$$
l(s, d)=\frac{1}{L(s, d)} \sum_{j=1}^{L(s, d)} n(s, d, j)
$$

where the sum in the numerator is taken over only those paths that share one or more links with the node $s$ to node $d$ shortest path being considered. It should be noted that the quantities $H(s, d), L(s, d), n(s, d, j)$ and $l(s, d)$ should only be computed for the source-destination node pairs $(s, d)$ for which the lightpath request traffic $\lambda_{s d}$ is non-zero. Assume that $\Omega$ is the set of such $s$ - $d$ node-pairs (with non-zero traffic). Then the weighting factor $x(i)$ for each node $i=1, \ldots, N$ is computed as follows -

1. Initialize $x(i)=0$ for $i=1, \ldots ., N$

2 . For each (shortest) path $s-d$ in the set $\Omega$, do the following For each intermediate node in path, excluding the source and the destination nodes, update $x(i)$ for node $i$ as

$$
x(i)=x(i)+\lambda_{s d} \frac{H(s, d)}{l(s, d)} \quad i=1, \ldots \ldots, N
$$

If $K$ is the number of nodes where converters are to be placed, then we choose these nodes as the ones with the $K$ highest values of $x(i)$.
The heuristic algorithm given above would not depend on $W$, the number of wavelengths on each fiber, as long as this value is the same for all the links. The algorithm may also be modified to handle the situation where $W$ is different for the different links.

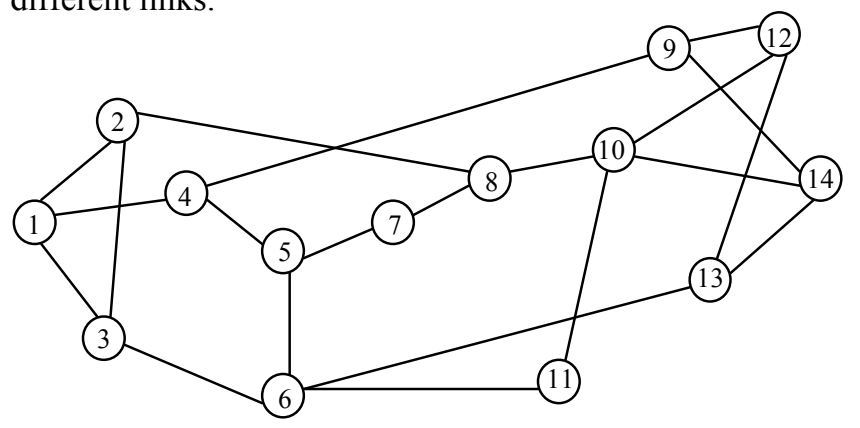

Fig. 1. NSFNET Network Topology

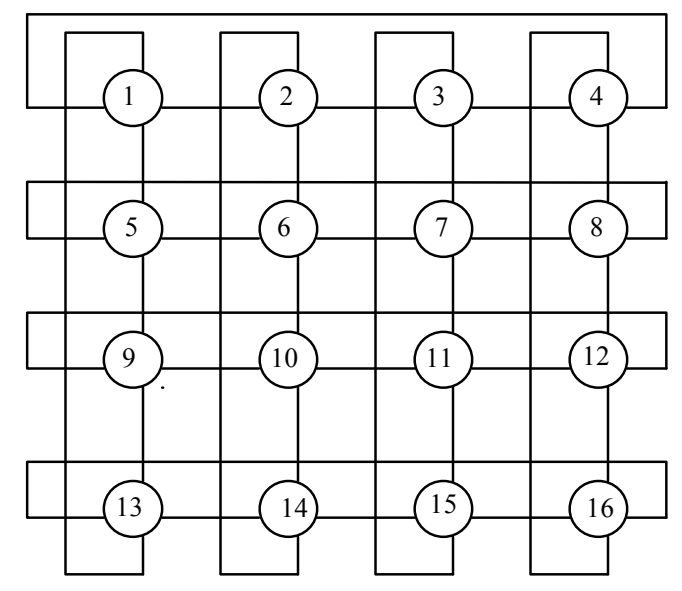

Fig. 2. A $4 \times 4$ Mesh-Torus Network

\begin{tabular}{|c|c|c|}
\hline $\mathrm{K}$ & Heuristic & Optimal \\
\hline 1 & 6 & 4 \\
\hline 2 & 4,6 & 4,6 \\
\hline 3 & $4,6,10$ & $4,6,8$ \\
\hline 4 & $4,6,8,10$ & $4,6,8,10$ \\
\hline 5 & $4,5,6,8,10$ & $4,5,6,8,10$ \\
\hline 6 & $4,5,6,8,9,10$ & $4,5,6,8,9,10$ \\
\hline 7 & $2,4,5,6,8,9,10$ & $2,4,5,6,8,9,10$ \\
\hline
\end{tabular}

Table 1: Indices of Converter Nodes for NSFNET

\begin{tabular}{|c|c|c|}
\hline $\mathrm{K}$ & Heuristic & Optimal \\
\hline 1 & 6 & 4 \\
\hline 2 & 6,11 & 6,11 \\
\hline 3 & $6,7,11$ & $6,7,11$ or $6,7,10$ \\
\hline 4 & $6,7,10,11$ & $6,7,10,11$ \\
\hline 5 & $6,7,10,11,12$ & $6,7,10,11,12$ \\
\hline 6 & $5,6,7,10,11,12$ & $5,6,7,10,11,12$ \\
\hline 7 & $5,6,7,9,10,11,12$ & $5,6,7,10,11,12,14$ \\
\hline
\end{tabular}

Table 2: Indices of Converter Nodes for 4x4 Mesh-Torus

In order to compare the results obtained with this heuristic, we compare the converter node placements obtained with the optimal placements given by [7]. This comparison has been 
done for the 14-node NSFNET and the $4 \times 4$ Mesh-Torus networks shown in Figs. 1 and 2. As in [7], we assume, uniform loading of 0.1 erlangs for the lightpath request traffic for each of the node pairs, i.e. $\lambda_{i j}=0.1 \forall i, j$ and unit mean holding times. The results for different values of $K$ are shown in Tables 1 and 2 for NSFNET and the Mesh-Torus network, respectively. For each value of $K$, these tables show the nodes at which the converters should be placed assuming that a node with converter will have full conversion capability. As may be seen from these tables, there are only very minor differences between the converter node placements obtained by us and those of [7]. Even in the case where differences do exist, we have verified through simulations that the network performances obtained using our placement and those of [7] are virtually identical..

We have also tested the heuristic algorithm for other irregular and regular networks and for both uniform and nonuniform traffic loading. It was generally observed that our heuristic results match closely those obtained from an optimal approach of [7] where ever that was applied. Simulation results also show that with full-converter nodes placed, as per the heuristic and/or optimal approaches, the overall lightpath blocking is less than that obtained with other placements.

\section{PATH-METRIC BASED AlgORITHM FOR DYNAMIC OPERATION OF WDM NETWORKS}

Lightpath establishment and termination would be dynamic random events in a WDM network where both route selection and wavelength selection for each link on the route will need to be done. A simple method [3] would be to identify a fixed shortest path between every node pair and provide heuristic algorithms for wavelength selection and converter usage along this path. Alternatively, a heuristic algorithm, as in [4], could be proposed for handling route and wavelength selection dynamically. A possible approach may be to do route selection as per the current graph of the network (in terms of links with free wavelengths) and then choose the wavelengths on each link on some rational basis (e.g. random or first-fit). Since the current graph will change dynamically, this algorithm may be difficult to apply in real-time. Moreover, wavelength selection and decisions on the nodes where converters are to be used will become difficult if nodes have only a limited numbers of wavelength converters, i.e. less than what would be required for full conversion.

In [11], we propose a simple path-metric based approach for making lightpath routing and wavelength and converter selection decisions quickly in a WDM network under dynamic traffic loading. We assume that, in general, a node has a limited number of converters available for wavelength conversion. We assume that blocked lightpath requests are lost and that a new lightpath request is routed and assigned wavelengths without affecting the existing lightpaths.

The strategy followed is to define a path-metric as a product of link and node metrics along each path. The metric is designed to give high values for good choices of path, wavelengths, and converter locations. A source-destination node pair will compute the path-metrics for all the possible choices that are feasible to establish a lightpath between them and establish the lightpath, which has the highest metric value. This procedure may be implemented in practice [11] using a mechanism where the source sends probe packets along each of the feasible paths from the source to the destination. Each of the probe packets collects data on wavelength and converter usage along its path allowing the destination to compute the required path-metrics and select the way in which the lightpath will be set up between the two nodes. Details on possible implementations are given in [11].

Along a given path, the path metric is computed as $W_{m} C_{m}$ where $W_{m}$ is the product of the metrics along each link of the $m^{t h}$ path and $C_{m}$ is the product of the metric for each node on the path where wavelength conversion is to be done. (Note that for nodes without converters, the corresponding metric term contributed to $C_{m}$ will be unity.) These link and node metrics are defined as follows.

$$
\begin{aligned}
W_{m} & =\prod_{k \in P_{m}}\left(1-\frac{W_{\text {used }}(k)}{W}\right) \\
C_{m} & =1 \quad \begin{array}{l}
\text { for WC path } \\
\text { for NWC path }
\end{array} \\
& =\prod_{n \in N_{m}} K_{n} \quad \text { if no wavelength conversion at } n^{\text {th }} \text { node } \\
K_{n} & =1 \quad \text { if conversion is done at the } n^{\text {th }} \text { node }
\end{aligned}
$$

The product terms of $W_{m}$ correspond to each of the links on the $m^{\text {th }}$ path/route being considered that has the set of links as $P_{m}$. As before, $W$ is the number of wavelengths on a link and we define $W_{\text {used }}(k)$ as the number of wavelengths already in use on the $k^{\text {th }}$ link in $P_{m}$. If a common wavelength can be found to establish a wavelength continuous (WC) path between the two end nodes, then $C_{m}$ is unity. Otherwise, $C_{m}$ is calculated as shown with the product terms corresponding to each of the nodes along the $m^{\text {th }}$ path that has the set of nodes as $N_{m}$. For a node with $C$ converters, we assume that $C_{u s e d}$ is the number of converters currently in use where the converters are used in a share per node fashion. The product term $K_{n}$ for the $n^{\text {th }}$ such node is unity if the node is such that it does not have any wavelength converters or if no wavelength conversion is done at that node. Otherwise, $K_{n}$ is the fraction of unused converters at the node. (Note that similar definition of $C_{m}$ may also be given for the share-per-link case.)

For a practical implementation of the path-metric based algorithm for dynamic operation, we assume that one primary path and an alternate path are predefined for each sourcedestination pair in the network. These, for example, may be obtained from the network graph using Dijkstra's shortest path algorithm or some similar strategy. More than two predefined paths may also be used though simulations [11] show that increasing the number of paths beyond two does not lead to a substantial performance improvement in typical 
networks.

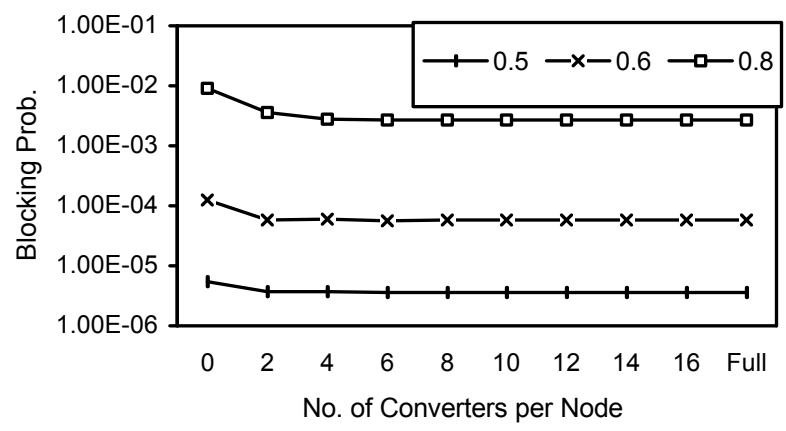

Fig. 3. Blocking Prob. vs. No. of Converters per Node ( $W=16$, share-per-link, NSFNET)

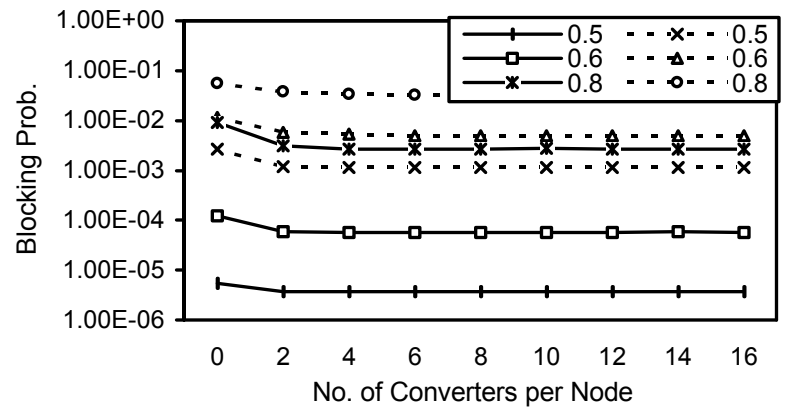

Fig. 4. Blocking Prob. vs. No. of Converters per Node ( $W=16$, share-per-node, NSFNET)

This path-metric based approach to dynamic operation of a WDM network has been extensively studied by us through a wide variety of simulations for different types of networks. As an example, we give in Fig. 3, the results obtained for the NSFNET network with $W=16$ and a variable number of converters at each node in the network and for lightpath traffic loads of $0.5,0.6$ and 0.8 . For purposes of comparison, we have also given the results obtained using the approach of [3]. Since the results of [3] are given for a share-per-link architecture, our results shown here are also for the same architecture. As can be seen from Fig. 3, the path-metric based approach performs considerably better than the scheme of [3] giving substantially lower values for the probability of blocking observed over the simulation interval. The corresponding share-per-node results are shown in Fig. 4. Note that "Full" in Fig. 4 indicates that the node has enough converters to convert all the wavelengths required. It may be observed that only a few converters are really needed at the nodes (each of the nodes in these cases) to improve the system's performance compared to the situation where the number of converters are zero and only WC lightpaths are allowed.

Other variations of this path-metric based approach have also been considered. A particularly useful variation is one where the two paths chosen for a node-pair are such that they have a minimum number of overlapping links, i.e. the alternate paths are "maximally disjoint". The results for this case are observed to be slightly better than that for the ones shown in Figs. 3 and 4. We have simulated this algorithm and its variations for a variety of networks (including NSFNET and ARPANET) for different values of $W$ and similar trends were observed in the results obtained.

\section{Dynamic Operation of Sparse Conversion Networks With Path-Metric Based Algorithm}

In this section, we consider the application of the pathmetric based algorithm for dynamic operation of a WDM network with sparse converter placement. For this, we assume that the converter nodes are selected based on the heuristic algorithm of Section II.

In Fig. 5, we compare the performance of the path-metric based scheme with other typical schemes suggested for lightpath routing when applied to the NSFNET network with sparse converter placement. The converter nodes selected are assumed to have full conversion capability for the simulation results shown in this figure and have been shown to vary between $K=0$ (no converter nodes) and $K=14$ (all nodes have converters). The simulations were done for a lightpath traffic load of 0.6 erlangs with $W=16$ for each fiber.

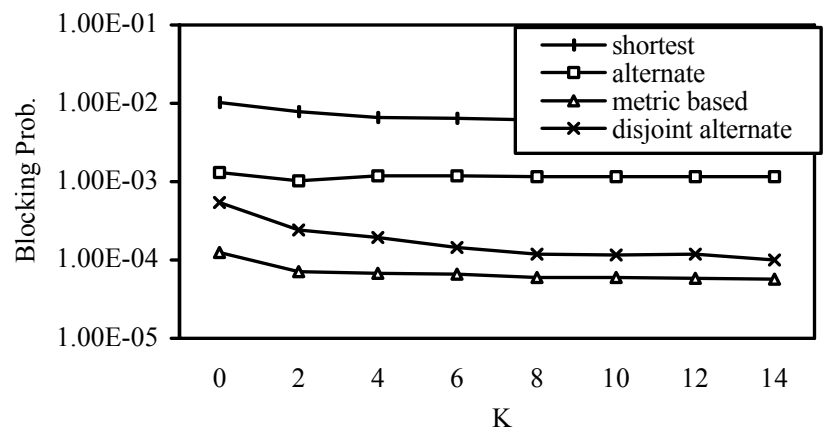

Fig. 5. Blocking Prob. vs. K (Traffic $=0.6$ erlangs, $W=16$, NSFNET)

The shortest path scheme uses only the (static) shortest path between the source and destination nodes for routing a lightpath request. For this, a WC lightpath is tried first. If a WC lightpath is not feasible, then a NWC lightpath is tried. A NWC path is then set up if wavelength resources are available in each link of the shortest path and appropriate converter resources are also available (at the intermediate converter nodes whose placement is decided by the heuristic converter placement algorithm) to do the required wavelength conversion as required. The alternate path scheme operates in the same manner as the shortest path scheme except that two paths are specified for each source-destination pair. The direct path is the shortest path (e.g. as obtained by Dijkstra's algorithm) which is searched for assigning a lightpath first. If a lightpath cannot be assigned on this path then the alternate path, which is the second shortest path from the network graph, is tried in a similar manner. The disjoint alternate path scheme is identical to the alternate path scheme except that the alternate path is chosen to be as disjoint as possible to the 
direct path. As may be seen from the figure, the shortest path scheme gives the worst performance, as there are no alternate paths available in this case. With two paths available per node-pair, the proposed path-metric based approach gives a significantly better performance than the other alternate path schemes. Similar results were obtained for different lightpath traffic values, $W$ and for different networks (e.g. ARPANET)

It is interesting to note that for each of the schemes, the performance improvement does not change significantly beyond a threshold value of $K$. This indicates that only a few converter nodes are really required to get good performance in the network. For higher values of $K$, the converter usage efficiency will be low and hence very little additional benefit will be obtained by increasing $K$ further. In order to improve converter usage efficiency, we can further limit the usage of converters to provide only limited conversion capability at the nodes selected for placing converters (these are selected as per our heuristic approach). Results for this for lightpath traffic loads of 0.6 and 0.8 are shown for the NSFNET topology in Fig. 6 where $C$ denotes the number of converters at a converter node. The converters are used in a share-pernode fashion with $W=16$ and $K=6$, i.e. six converter nodes at the nodes labeled 4,5,6,8,9, and 10 of Fig. 1.

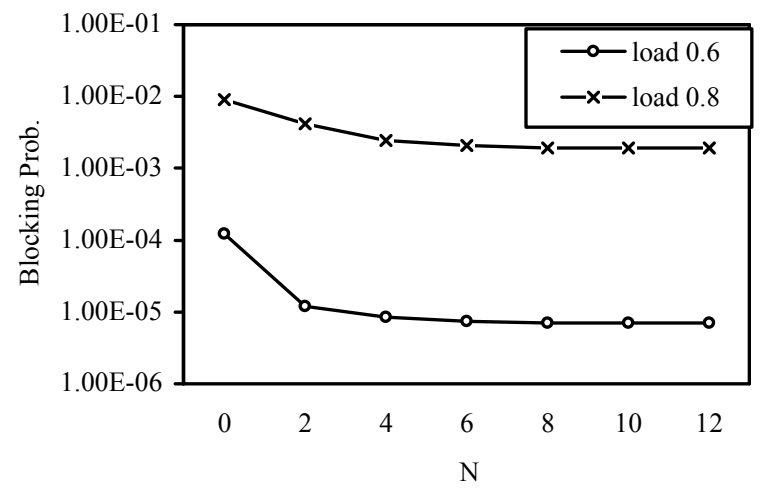

Fig. 6. Blocking Prob. vs. $C$ ( $W=16, K=6$, NSFNET)

As is typical of WDM systems with lightpath conversion [2]-[4], we observe that only a few converters (i.e. small values of $C$ ) are needed at the converter nodes to give most of the benefits that one can obtain from wavelength conversion. Similar trends are also seen for other network topologies (e.g. ARPANET), when the parameters $W$ and $K$ and the lightpath traffic load are varied. Considering this effect with our earlier observation on the efficacy of sparse converter placement using our suggested heuristic implies that these algorithms may be combined to design and operate a WDM network efficiently even with a few wavelength converters. The heuristic placement algorithm for the converter nodes may be used to efficiently design the WDM network where only a few nodes (i.e. $K$ ) are actually equipped with wavelength converters. Even if the converter nodes have only a limited number of converters, the network can still be efficiently operated with the path-metric based approach.

\section{CONCLUSIONS}

We present a simple heuristic algorithm for converter node placement, which gives results that are observed to be as good as that obtained through optimization procedures. A path-metric based algorithm has also been proposed to efficiently operate a WDM network with limited or full conversion capability at the converter nodes. Using the two algorithms together will allow WDM networks, which require very few converters but can give results that are substantially similar to that of networks with full conversion capability at all its nodes. Since wavelength converters are costly, this approach will reduce the cost of the overall network without significantly sacrificing on its performance.

\section{REFERENCES}

[1] R. Ramaswami and K. Sivarajan, Optical Networks: A Practical Perspective, Morgan Kaufmann Publishers, 1998.

[2] J. M. Yates et al, "Wavelength converters in dynamically reconfigurable WDM networks," IEEE Communication Surveys, Second Quarter 1999, pp. 2-15.

[3] Gangxiang Shen et al, "Operation of WDM networks with different wavelength conversion capabilities," IEEE Communications Letters, July 2000, pp. 239-241.

[4] Gangxiang Shen et al, "Heuristic algorithms for efficient lightpath routing and wavelength assignment," Computer Communications, vol. 24 (2001), February 2001, pp. 364-373.

[5] S. Subramaniam et al, "On the optimal placement of wavelength converters in wavelength routed networks," IEEE INFOCOM, 1998, pp. 902-909.

[6] G. Xiao, Y Leung, "Algorithms for allocating wavelength converters in all-optical networks," IEEE/ACM Trans. on Networking, vol. 7, no. 4, August 1999, pp. 545-557.

[7] S. Thairajan, A Somani, "An efficient algorithm for optimal wavelength converter placement on wavelengthrouted networks with arbitrary topologies," IEEE INFOCOM, 1999, pp 916-923.

[8] H. Harai et al, "Allocation of wavelength convertible nodes and routing in all-optical networks," Proc. SPIE All Optical Communication Systems: Architecture, Control and Network Issues III, vol. 30, Ocober 1997, pp 277-287.

[9] C.Vijayanand et al, "Converter placement in all-optical networks using genetic algorithms," Computer Communications, vol. 23, 2000, pp. 1223-1234.

[10]Bhoomika Popat, "Heuristic converter placement algorithm and study of its effects on sparse WDM networks," M.Tech. Thesis, Dept. of Electrical Engg., I.I.T., Kanpur, India, February 2001.

[11]A.N.V.B. Raju, "Path-metric based routing for dynamic operation of WDM networks with varying conversion capabilities," M. Tech. Thesis, Dept. of Electrical Engg., I.I.T., Kanpur, India, March 2001. 